\title{
Origins Space Telescope integration and testing
}

\author{
Susanna Petro* \\ National Aeronautics and Space Administration, Goddard Space Flight Center, \\ Greenbelt, Maryland, United States
}

\begin{abstract}
I describe the plans, flows, key facilities, test beds, pathfinders, simulators, and ground support equipment that could be used to fully integrate, functionally test, and qualify the Origins Space Telescope (Origins). The Origins observatory consists of the spacecraft bus module and the cryogenic payload module, which comprises the telescope and three science instruments. The telescope is a three-mirror anastigmat and is composed of four mirrors: three with optical power (the elliptical primary, hyperbolic secondary, and elliptical tertiary mirrors) and a flat field-steering mirror. The three science instruments spanning the wavelength range 2.8 to $588 \mu \mathrm{m}$ provide the powerful new spectroscopic and imaging capabilities required to achieve the scientific objectives. The Origins Survey Spectrometer uses six gratings in parallel to take multibeam spectra simultaneously across the $25-$ to $588-\mu$ m window through long slits enabling deep three-dimensional extragalactic surveys. The far-IR imager/polarimeter provides imaging and polarimetric measurement capabilities at 50 and $250 \mu \mathrm{m}$. Its fast mapping enables rapid follow-up of transient or variable sources and efficient monitoring campaigns. The mid-infrared spectrometer simultaneously provides spectroscopy over 2.8 to $20 \mu \mathrm{m}$ with exquisite stability and precision ( $<5 \mathrm{ppm}$ between 2.8 and $10 \mu \mathrm{m},<20 \mathrm{ppm}$ between 11 to $20 \mu \mathrm{m}$ ). All the instruments are delivered for integration and test fully qualified and calibrated. The integration and test program implemented at each level of assembly is discussed as well as the separation of thermal vacuum testing between the hot and cold zones of the observatory. (c) The Authors. Published by SPIE under a Creative Commons Attribution 4.0 Unported License. Distribution or reproduction of this work in whole or in part requires full attribution of the original publication, including its DOI. [DOI: 10 .1117/1.JATIS.6.4.041502]
\end{abstract}

Keywords: Origins; optics; cryogenic telescope; integration and test; decadal survey.

Paper 20055SS received May 20, 2020; accepted for publication Sep. 1, 2020; published online Oct. 9, 2020.

\section{Introduction}

Portions of this paper appear in the Origins Study Final Report. ${ }^{1}$

The Origins Space Telescope (Origins) traces our cosmic history, from the formation of the first galaxies and the rise of metals to the development of habitable worlds and present-day life. Origins does this through exquisite sensitivity to infrared radiation from ions, atoms, molecules, dust, water vapor, and ice, and observations of extra-solar planetary atmospheres, protoplanetary disks, and large-area extragalactic fields. Origins operates in the wavelength range 2.8 to $588 \mu \mathrm{m}$ and is more than 1000 times more sensitive than its predecessors due to its large, cold $(4.5 \mathrm{~K})$ telescope and advanced instruments.

\section{Origins Overview}

Origins will trace the history of our origins from the time dust and heavy elements permanently altered the cosmic landscape to present-day life. ${ }^{1}$ How did galaxies evolve from the earliest galactic systems to those found in the universe today? How do habitable planets form? How common are life-bearing worlds? To answer these questions, Origins will operate at mid- and far-infrared wavelengths and offer powerful spectroscopic instruments and sensitivity three orders of magnitude better than that of Herschel, the largest telescope flown in space to date.

*Address all correspondence to Susanna Petro, E-mail: Susanna.petro-1@nasa.gov 


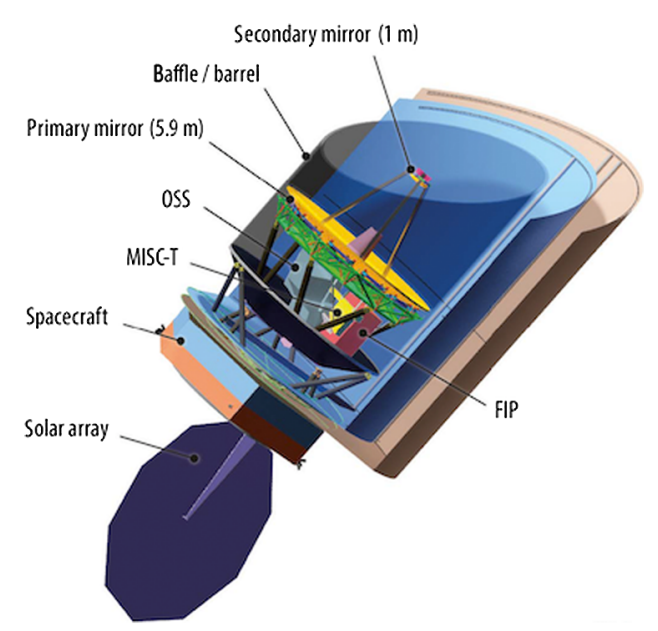

Fig. 1 The Origins cutaway view shows the Origins instrument locations and major elements of the flight system.

Origins has a Spitzer-like architecture (Fig. 1) and requires only a few simple deployments to transform from launch to operational configuration. The Origins observatory consists of the spacecraft bus module (SBM) and the cryogenic payload module (CPM), which comprises the telescope and three science instruments. The telescope is a 5.9-m diameter, cryocooled to $4.5 \mathrm{~K}$, three-mirror anastigmat and is composed of four mirrors: three with optical power (the elliptical primary, hyperbolic secondary, and elliptical tertiary mirrors) and a flat field-steering mirror.

The optical system ${ }^{2}$ launches in its operational configuration, requiring no mirror, barrel, or baffle deployments after launch, but the design allows for mirror segment alignment on orbit to optimize performance. The solar panel, communication antenna, and telescope cover deployment mechanisms have extensive heritage. The two-layer sunshield deployment relies on stored energy in flexible rods to pull the shield material into its desired shape, and telescoping arms to place each shield at the intended distance from the cold shield. This simple deployment sequence can be tested on the ground in existing facilities.

The three science instruments ${ }^{3}$ spanning the wavelength range 2.8 to $588 \mu \mathrm{m}$ provide the powerful new spectroscopic and imaging capabilities required to achieve the scientific objectives. The Origins Survey Spectrometer (OSS) uses six gratings in parallel to take multibeam spectra simultaneously across the 25 - to $588-\mu \mathrm{m}$ window through long slits enabling deep threedimensional extragalactic surveys. The far-IR imager/polarimeter (FIP) provides imaging and polarimetric measurement capabilities at 50 and $250 \mu \mathrm{m}$. Its fast mapping enables rapid follow-up of transient or variable sources and efficient monitoring campaigns. The Mid-Infrared Spectrometer Camera Transit Spectrometer simultaneously provides spectroscopy over 2.8 to $20 \mu \mathrm{m}$ with exquisite stability and precision $(<5 \mathrm{ppm}$ between 2.8 and $10 \mu \mathrm{m},<20$ ppm between 11 and $20 \mu \mathrm{m})$.

Origins thermal performance is well understood. The observatory architecture consists of a cold zone with telescope and instruments operating at cryogenic temperatures and a hot zone with the spacecraft bus (Fig. 2). The cryothermal system design leverages Spitzer experience and technology developed for James Webb Space Telescope (JWST) and Hitomi. Four current stateof-the-art cryocoolers will cool the telescope to $4.5 \mathrm{~K}$, with $100 \%$ margin in heat lift capacity at each temperature stage.

The mirrors do not require time-consuming "cryonull" figuring because the telescope is diffraction limited at $30 \mu \mathrm{m}$. All of the telescope's mirrors and mirror segments can be diamondturned and rough-polished to the required precision in existing facilities. The JWST primary mirror segment actuator design is adopted to allow the Origins primary mirror segments to be adjusted in space in three degrees of freedom (tip, tilt, and piston), enabling final alignment during commissioning.

The next generations of launch vehicles, including the National Aeronautics and Space Administration's (NASA) Space Launch System, SpaceX's Starship, and Blue Origin's 7-m 


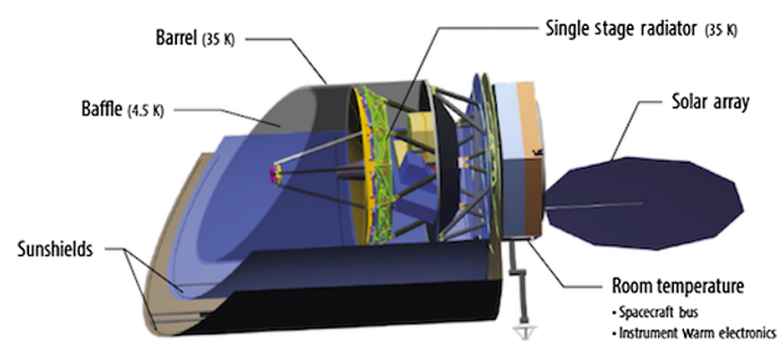

Fig. 2 Origins temperature zones are designed to maximize cryocooling efficiency while ensuring a 4.5-K environment for the telescope and instruments. The instruments are housed in the 4.5-K zone beneath the primary mirror.

New Glenn, have much larger payload fairings than the 5-m diameter fairings available today, enabling the launch of a large diameter telescope that does not need to be folded and deployed. Origins will operate in a quasi-halo orbit around the Sun-Earth L2 point. The observatory is robotically serviceable, enabling future instrument upgrades and propellant replenishment to extend the mission beyond its 10 -year design lifetime.

\section{Integration and Test (I\&T) Overview}

The major subassemblies of the fully assembled Origins observatory are defined as follows.

- Instruments, consisting of the following: Spectrometer (OSS), FIP, and MID-IR imager spectrometer/coronagraph (MISC). The three instruments are delivered to I\&T fully qualified and calibrated.

- Telescope, consisting of the following: primary mirror segments, secondary mirror, aft optics, cryocoolers, inner optical baffle, outer barrel, sunshield structure and deployment mechanism, protective cover, and telescope harness. Access doors are located around the barrel for instrument access and integration. The optical elements of the 5.9-m diameter telescope are shown in Fig. 3.

- CPM, consisting of the following: structural/mechanical, telescope, OSS, FIP, MISC, cryocooler interface hardware, cold electronics, CPM harness, and inner and outer sunshield materials. The CPM components exploded view is shown in Fig. 4. The CPM dimensions are shown in Fig. 5.

- SBM, consisting of the following: structural/mechanical, attitude control system, propulsion, propellant and gas, command and data handling, communications, power, instrument warm electronics, antennas, cryocoolers, solar array, and spacecraft harness. The SBM dimensions are shown in Fig. 6.

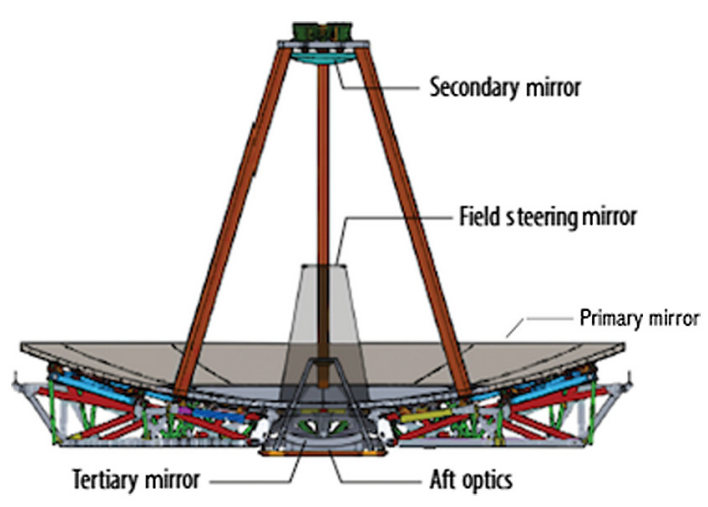

Fig. 3 Telescope optical elements. Aft optics structure mounts to the Primary Mirror Backplane Support Structure and contains the tertiary mirror and field steering mirror. 


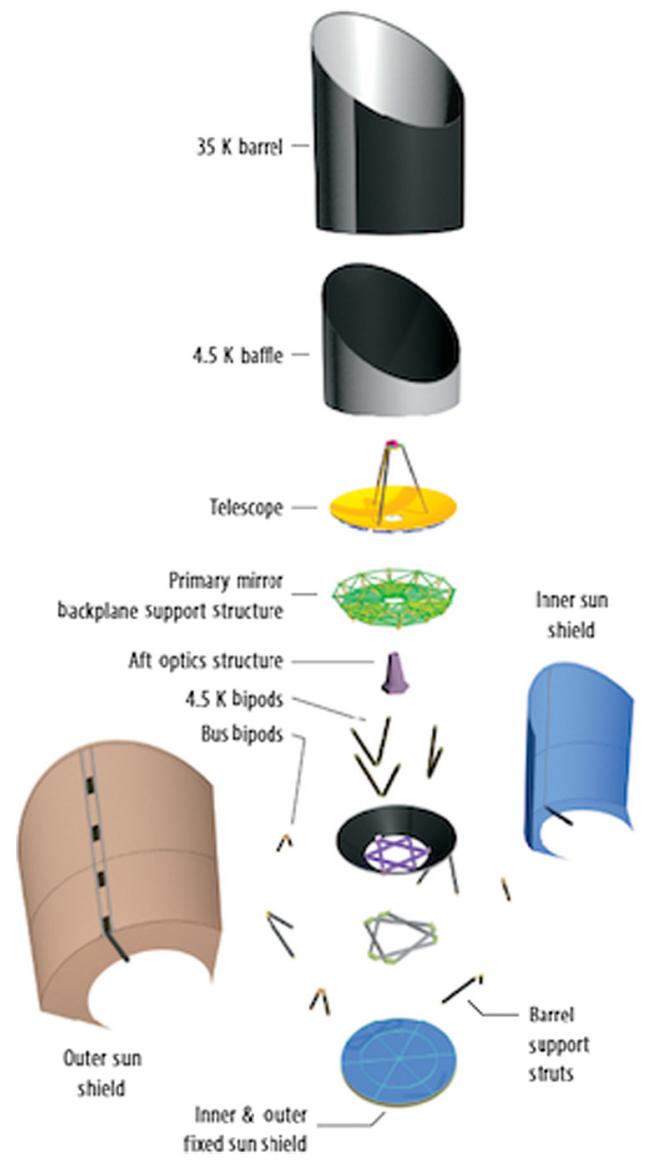

Fig. 4 Origins CPM components exploded view shows the Origins configuration. Instruments are not shown in this figure. ${ }^{1}$

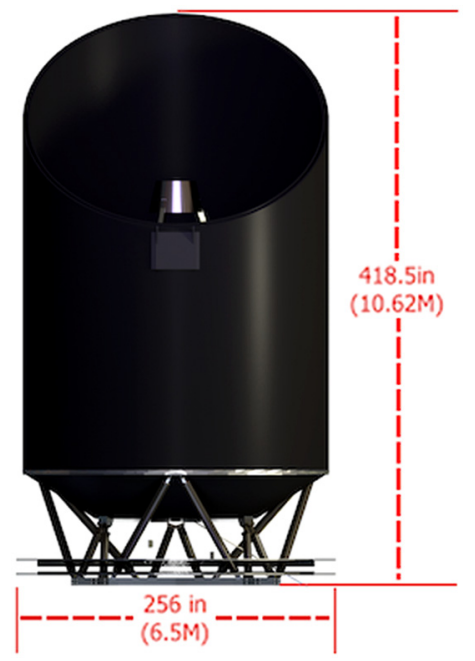

Fig. 5 Origins CPM dimensions.

- Observatory, consisting of the following: CPM, SBM, and observatory harness. The observatory dimensions are shown in Figs. 7 and 8.

The summary level I\&T flow is shown in Fig. 9. Each of these phases is further detailed in the subsequent sections below. 
Petro: Origins Space Telescope integration and testing

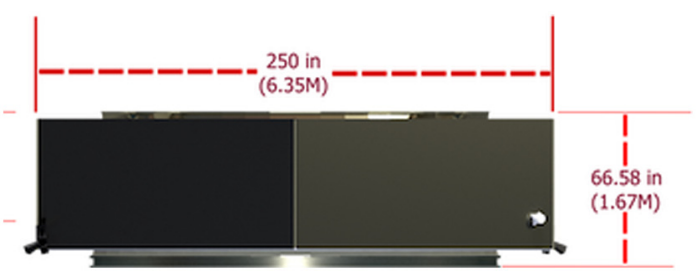

Fig. 6 Origins SBM dimensions.

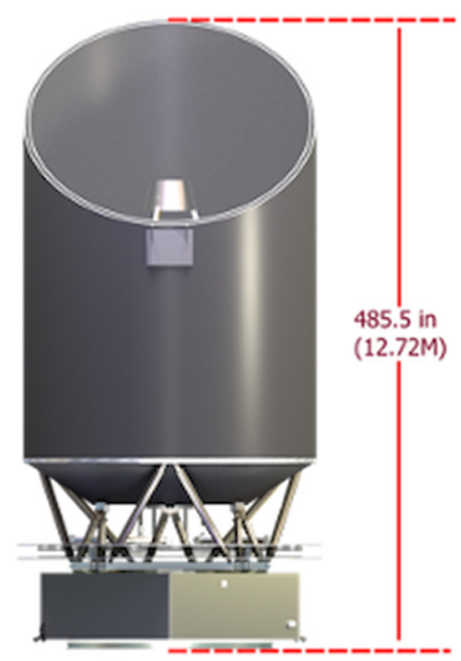

Fig. 7 Origins observatory dimensions (front).

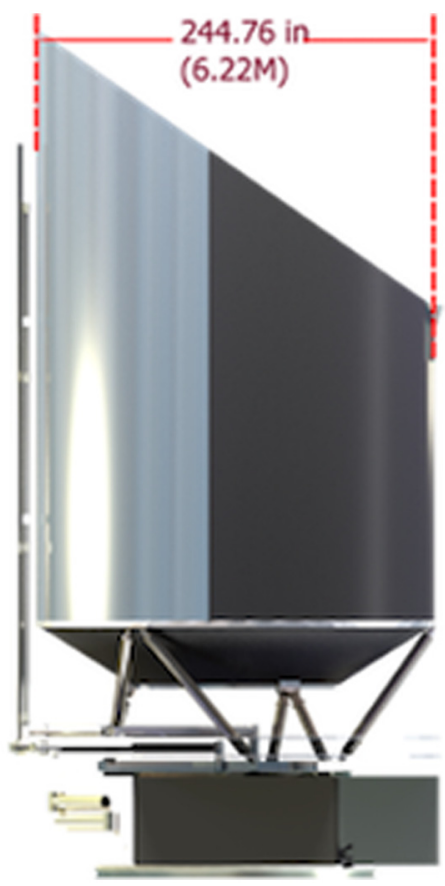

Fig. 8 Origins observatory dimensions (side). 


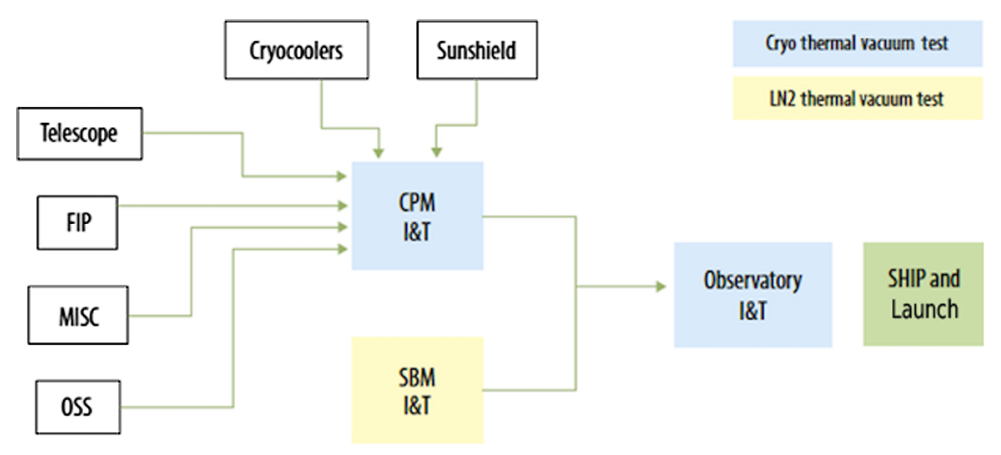

Fig. 9 Origins I\&T summary flow for CPM and SBM. Each of these phases is further detailed in subsequent sections.

Verification of Origins thermal performance by test with the observatory fully assembled is far more challenging than a typical spacecraft design due to the large size of the primary mirror and sunshield and the wide operational temperature gradient. As a result, Origins will perform separate thermal vacuum tests of the cold and hot zones of the observatory. Protoflight level environmental tests will be performed both at the element level and at the observatory level. Simulation of missing interfaces during tests of the lower levels of assembly and testing of various nonflight models will provide the data for final analysis and verification of the overall observatory performance. ${ }^{4}$

Commonality of test personnel and products across each level of assembly, including test sets, command and telemetry system/database, procedures, and automated test scripts will provide test consistency across levels of assembly, enable test product reuse, and reduce cost and schedule risk. Similarly, mechanical ground support equipment (GSE) for flight hardware lifting, rotations, and deployments will be also used for mechanical operations and testing.

Contamination control is another key driver of Origins I\&T processes. External optic cleanliness can only be maintained through the use of the International Standards Organization Class 7 facilities (class $10 \mathrm{~K}$ ) and personnel protocols throughout all phases of I\&T.

Testbeds, pathfinders, developmental test articles, mock-ups, and simulators will reduce risk, provide a venue for tests that cannot be performed on the flight hardware, or simulate flight interfaces that are not available for a particular flight test. Origins will utilize many articles of this type in addition to the flight I\&T program.

An engineering model test bed (EMTB) will integrate engineering model electrical units together with a flight-like harness and a duplication of the I\&T electrical test environment. The EMTB objective is to perform validation of flight software as well as electrical compatibility of observatory electronics. EMTB is a shared resource between systems engineering for flight design objectives as well as I\&T for test process demonstration objectives.

Various configurations of spacecraft simulators will be built and delivered to lower levels of assembly to provide a flight-like command and telemetry interface for "test as you fly" before the CPM is ever mated with the flight spacecraft. Spacecraft simulators used in conjunction with a common command and telemetry system and database at all levels of assembly facilitate reuse of test products at each level of assembly.

\section{Telescope I\&T}

Assembly integration and testing of the telescope can be performed in a number of facilities in the United States. One possible venue for mirror assembly is at the Goddard Space Flight Center (GSFC) in the Spacecraft Systems Development and Integration Facility (SSDIF) shown in Fig. 10, which was also used for JWST telescope assembly.

Telescope I\&T will start with assembly of the structural subassemblies. A rollover fixture is used to orient the structure as required for assembly access. Then optics installation starts. The Aft Optics, all primary mirror assemblies, and the secondary mirror are installed and aligned to 


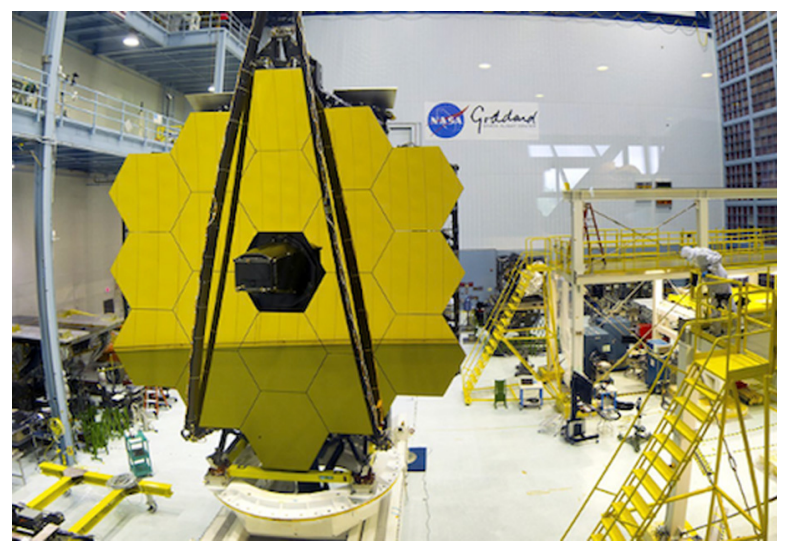

Fig. 10 JWST telescope assembled in the SSDIF at NASA/GSFC.

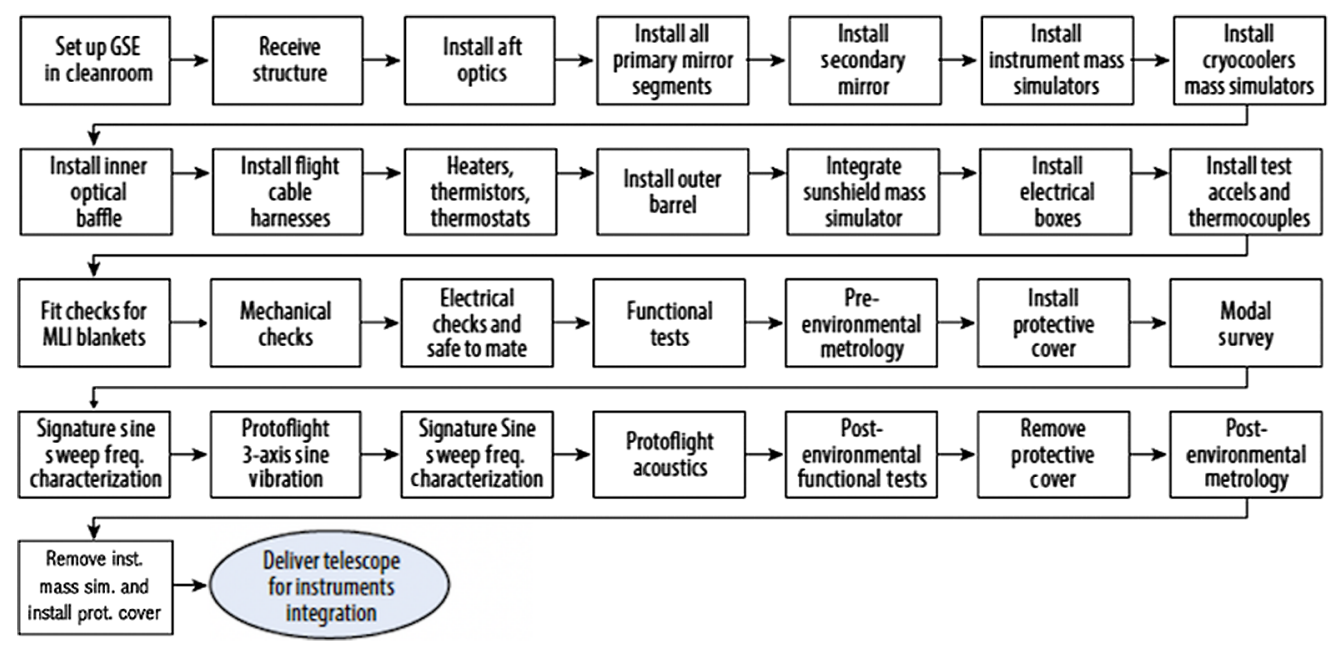

Fig. 11 Telescope I\&T summary flow. GSE stands for ground support equipment.

the structure. Then the instrument mass simulators, cryocoolers, inner optical baffle, telescope harness, outer barrel, sunshield structure and deployment mechanism, electrical boxes, and protective cover are integrated. The completed telescope assembly is then ready for the mechanical test program (modal survey, signature sine sweep frequency characterization, and protoflight three-axis sine vibration), followed by one more signature sine sweep frequency characterization and protoflight acoustics. Alignment is performed before and after these tests. The scope of this test program is to verify the stability of the mirrors, structures, and mechanisms. After the telescope testing is complete, the instrument mass simulators are removed and the telescope is ready for integration with the instruments. The Telescope I\&T summary flow is shown in Fig. 11.

\section{Cryogenic Payload Module I\&T}

The primary objective of the integration and test program is to successfully deliver a fully tested and verified CPM that meets all functional requirements and performs as designed when placed on the SBM to create the Origins observatory.

The CPM integration can be accommodated at GSFC in the SSDIF. Although specific locations are given to perform various tests, these are meant to be examples only that show one way in which the tests can be accomplished. Other facilities at different locations in the US may also be used for these tests. Specialized GSE development is required for the integration of all 


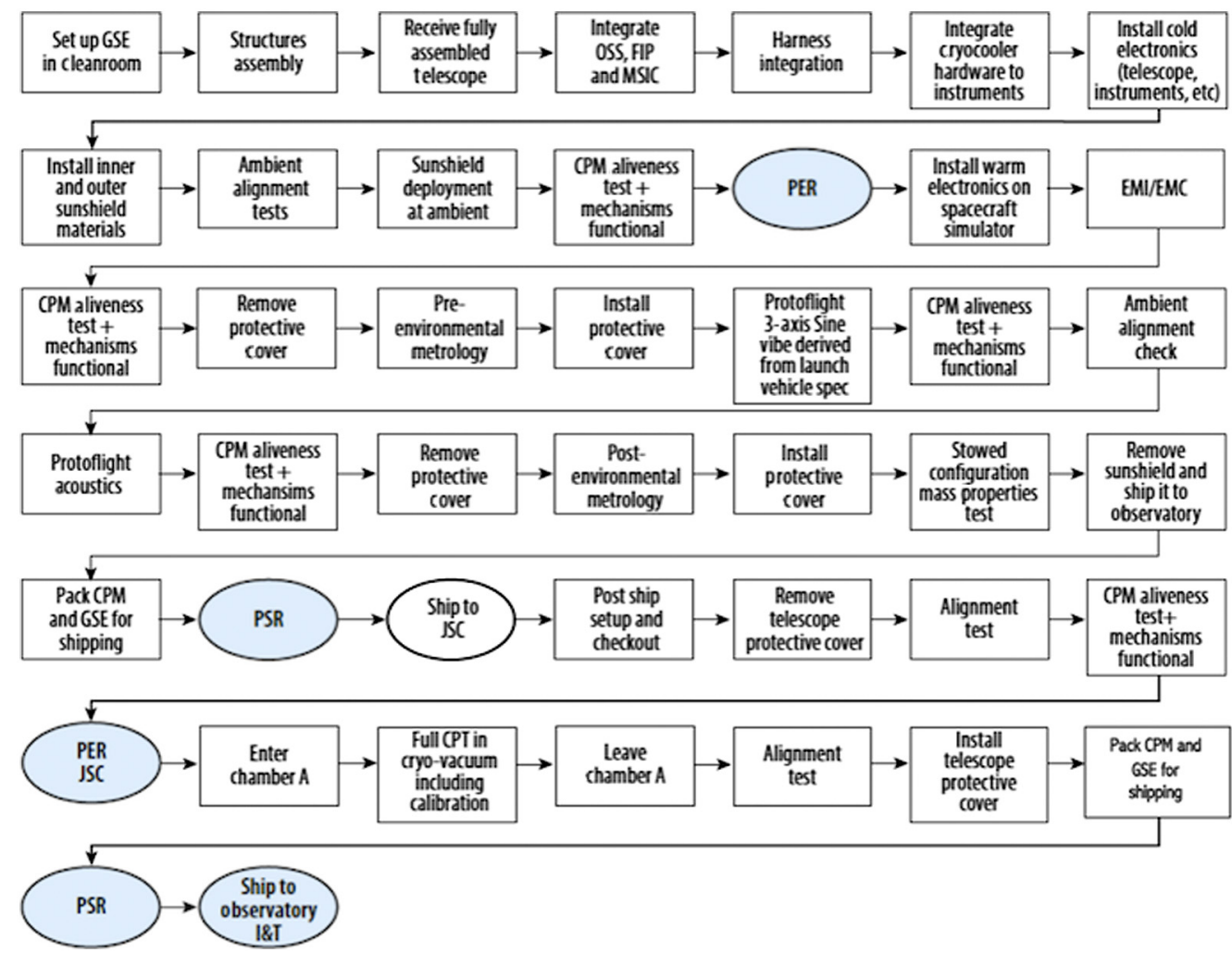

Fig. 12 CPM I\&T summary flow. It includes the milestones reviews: Pre-Environmental Review (PER) and Pre-Ship Review (PSR).

components. Tests to verify the optical and thermal performance of the GSE will be included in the I\&T plan. This approach has been used on JWST for which two optical GSE tests were performed to demonstrate optical test equipment and methodologies. The CPM I\&T summary flow is shown in Fig. 12.

After ambient integration of the fully assembled telescope with instruments, electromagnetic interference (EMI)/electromagnetic compatibility (EMC) testing will be performed. This test will be followed by protoflight vibration and protoflight acoustics tests. Aliveness test and ambient metrology will also be performed pre- and postmechanical testing to ensure the hardware remains aligned. The environmental test campaign (except for the cryogenic thermal vacuum test) can be accommodated at GSFC. The GSFC acoustic chamber is shown in Fig. 13. EMI/EMC testing requires using a customized tent.

The team will design and manufacture a specialized in-plant transporter (IPT) to move the flight hardware from the cleanroom to the vibration and acoustics facility. The IPT will include a portable clean tent around the CPM to provide the required cleanliness. The sunshield deployment test will be conducted at this point. After the mechanical tests, the team will perform a mass properties test. The hardware is then prepared for transportation and transported to the Johnson Space Center (JSC) by a shipping container whose size is compatible with the Super Guppy or Beluga aircraft. Note: the transporter was called Space Telescope Transportation Air, Road and $\mathrm{Sea}^{4}$ for JWST. Upon arrival at JSC, the flight CPM is removed from the transporter and prepared for installation into Chamber A (shown in Fig. 14).

Preparations include telescope protective cover removal, GSE sensor installation, metrology, and ambient functional testing. A 4.5-K GSE cover will be installed to reduce the stray light from the $11 \mathrm{~K}$ shroud to the instruments. This cover will be cooled by a 4-K GSE cryocooler, similar in concept to the GSE cooler used to cool the mid-infrared instrument during the JWST Optical Telescope Element and Integrated Science Instrument Module (OTIS) cryogenic tests. Once installed into chamber A, the cryogenic testing of the CPM will begin. In general, the objective 


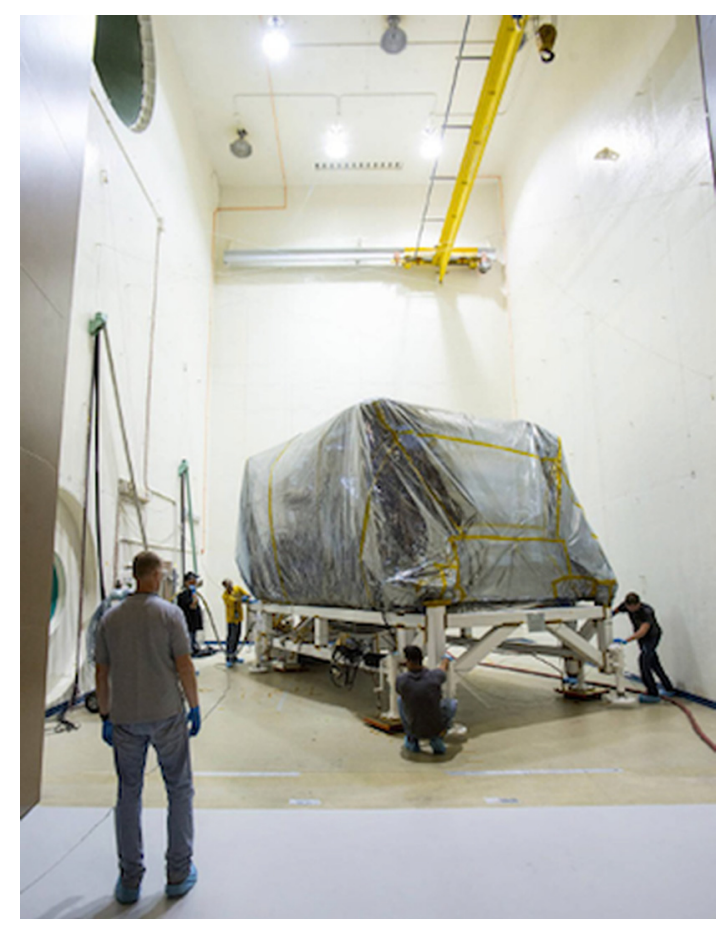

Fig. 13 The GSFC acoustic chamber can easily accommodate testing the Origins CPM. Note that this chamber is not large enough for the fully assembled Origins observatory. This test will be performed at the JSC or another suitable facility.

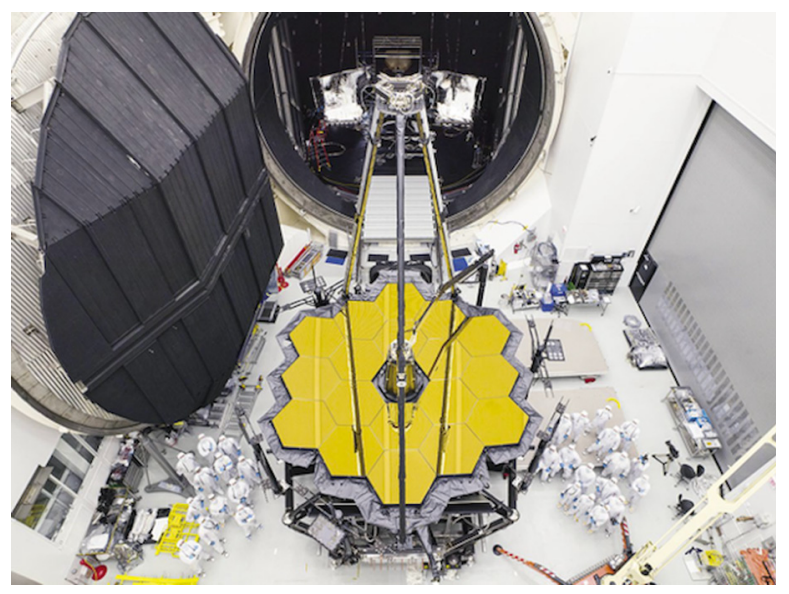

Fig. 14 JSC chamber A supports Origins "test as you fly." The JWST OTIS is shown rolling into the JSC chamber A. Chamber A and its helium cooled shroud provides a perfect environment for thermal verification of Origins with and without the spacecraft.

of the cryogenic vacuum test is to verify the CPM level requirements in the conditions of the expected flight environment, with emphasis on optical measurements that can be performed in this test configuration. This is the first time that the integrated instruments will be fully operational at their flight temperature. The optical tests ${ }^{1}$ will verify the CPM system optical workmanship and provide optical test data to support the integrated telescope modeling used to predict flight optical performance. After the testing, the CPM is then removed from the chamber and prepared for transportation. The hardware is transported to the integration facility where it will be mated to the SBM to create the Origins observatory. 


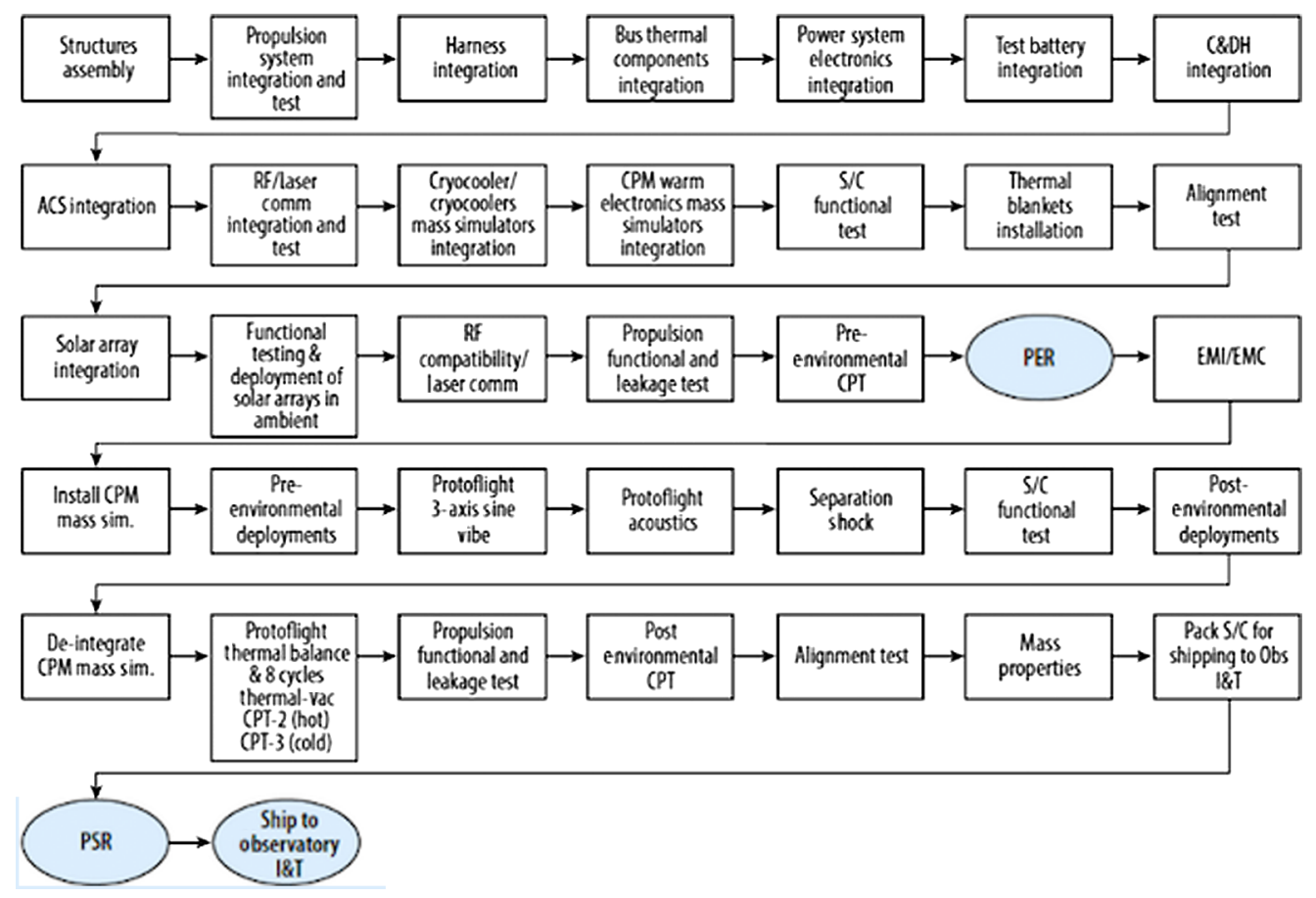

Fig. 15 SBM I\&T summary flow. This includes the milestones reviews: PER and PSR. S/C stands for SBM. CPT stands for comprehensive performance test.

\section{Spacecraft Bus Module I\&T}

The SBM I\&T consists primarily of the spacecraft bus (which includes the typical spacecraft bus subsystems of power, attitude control, communications, command, and data handling), solar array, cryocoolers mass simulators, and instrument warm electronics mass simulators. The SBM primary structure is delivered to I\&T already qualified. The objective of the SBM I\&T is to deliver a fully verified and tested element that meets all the functional requirements prior to integration with the CPM at the start of the observatory I\&T. The SBM I\&T summary flow is shown in Fig. 15.

The SBM integration begins with the propulsion system integration followed by the harness integration and the assembly of thermal components and electronics. Validated flight software is delivered and utilized throughout the remainder of I\&T. A complete electrical integration of all bus mounted electronics is performed in a "flat sat" configuration. Flight electrical equipment that will be mounted to the bus structure is temporarily mounted to a ground support table to enable full flight electrical integration of the entire complement of busmounted equipment. Electrical system integration issues are typically discovered during "flat sat" I\&T, which is performed independent of the bus structure critical path. Major tests during SBM I\&T include an initial ambient baseline electrical comprehensive performance test (CPT), alignments, ambient deployments, and radio frequency compatibility/laser communications test. The environmental test campaign includes: EMI/EMC, protoflight three-axis sine vibration, protoflight acoustics, protoflight thermal balance and thermal vacuum, and propulsion functional and leakage tests. These tests could be done at GSFC using the T 4000-3 shaker (shown in Fig. 16), the large EMI/EMC chamber and control console (shown in Figs. 17 and 18), the Mass Properties Measurement Facility (shown in Fig. 19), and the space environment simulator (SES, shown in Fig. 20). There are many other facilities in the US where these tests can be done. CPM interfaces are electrically simulated during functional and environmental testing. A fully tested and verified SBM is ready for mating with the CPM in observatory I\&T. 


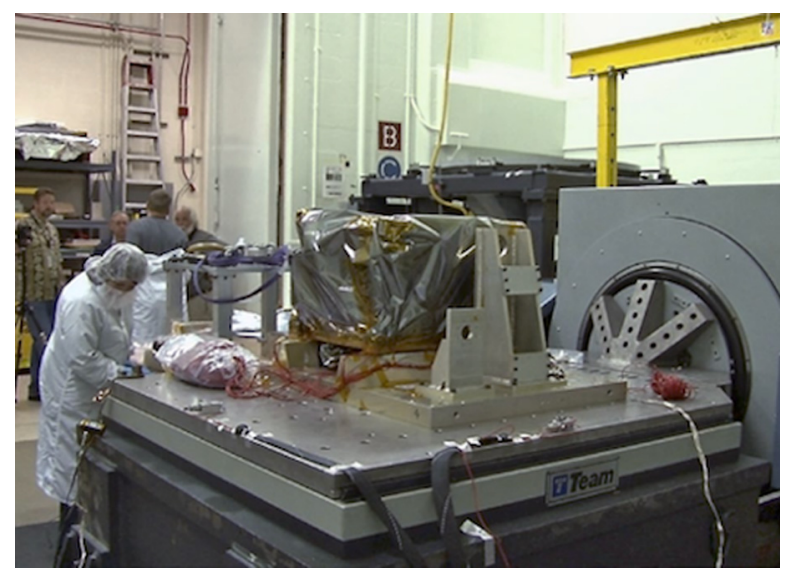

Fig. 16 Facility 410-Unholtz-Dickie T4000-3 Shaker.

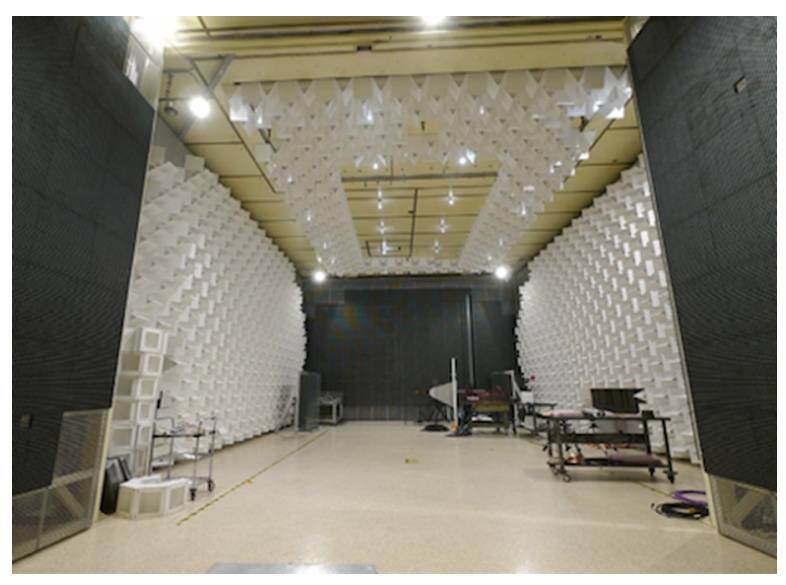

Fig. 17 Large EMC shielded enclosure.

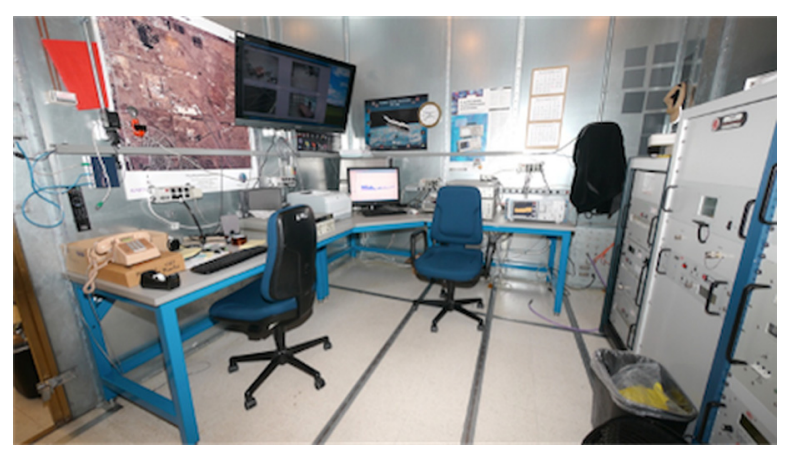

Fig. 18 Large EMC control console.

\section{Observatory I\&T}

The observatory I\&T integrates the fully qualified CPM with the fully qualified SBM. Facilities already exist to perform this test campaign. Telescope and instruments warm flight electronics and flight cryocoolers are integrated into SBM. Inner and outer sunshield supports 


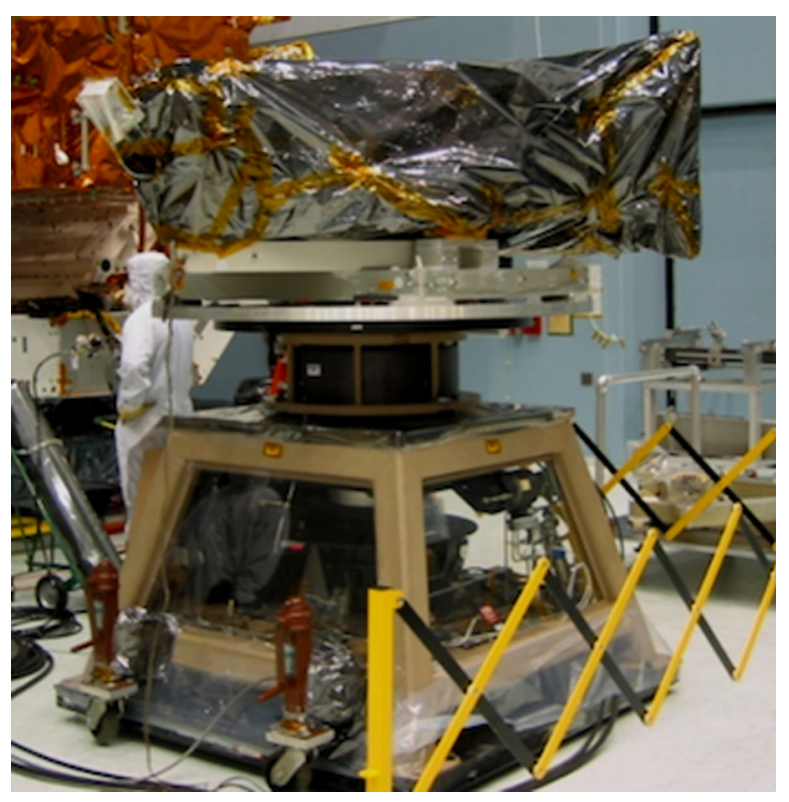

Fig. 19 Mass Properties Measurement Facility.

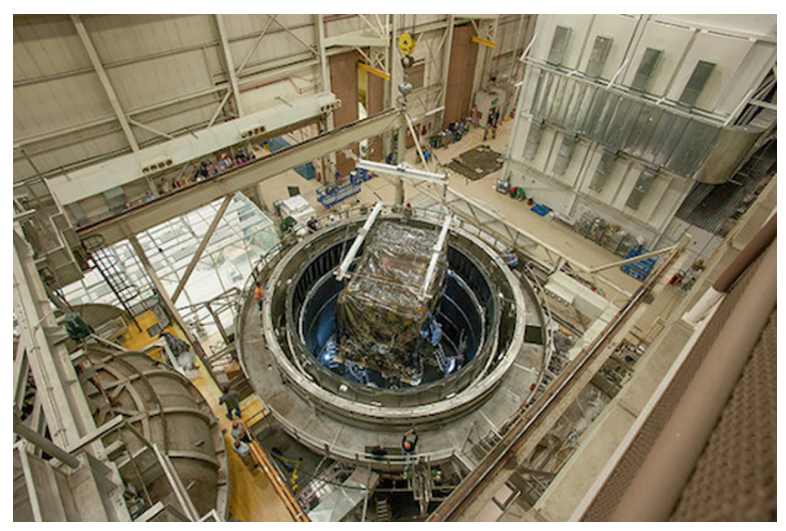

Fig. 20 SES chamber.

and material are integrated into the CPM. The CPM and SBM are integrated. The harness is integrated. Alignment is performed. Full electrical and software compatibility across the interface has been previously validated. Testing at CPM and SBM levels will also utilize high-fidelity simulators to validate those interfaces through ambient and protoflight level environmental testing. The observatory environmental campaign consists of aliveness tests, EMI/EMC, acceptance three-axis sine vibration, shock, and mass properties that can be done with the variable center of gravity mechanism, already used with JWST and shown in Fig. 21.

Then the observatory will be shipped to JSC for acceptance acoustics. Once this test is completed, the observatory will be moved into chamber A where a sunshield deployment will be performed in vacuum, followed by a CPT, end to end data test (hardline), and special tests (loss of power, etc.). At this point, the observatory will be removed from chamber A. Then a functional test, laser communications verification, alignment, and leak test will be performed. Finally, the observatory is stowed in launch configuration and prepared for transportation to the launch site. The observatory I\&T flow is shown in Fig. 22. 


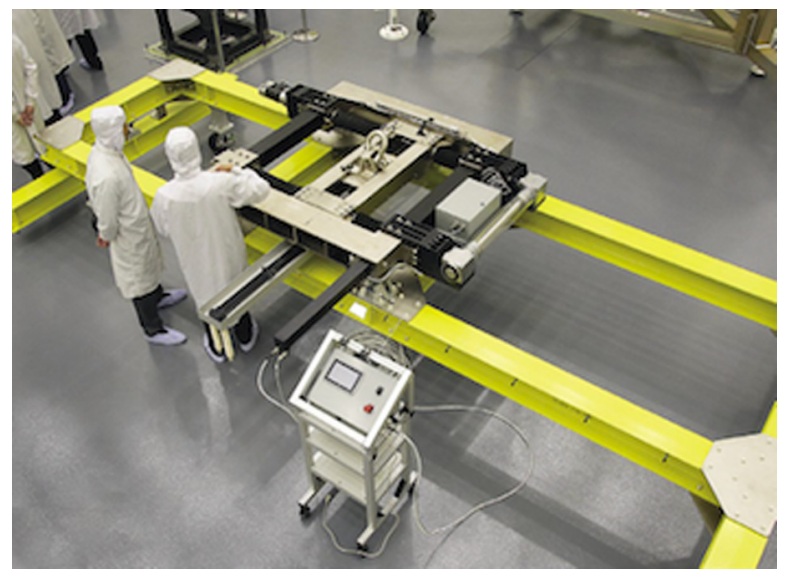

Fig. 21 Variable Center of Gravity Mechanism.

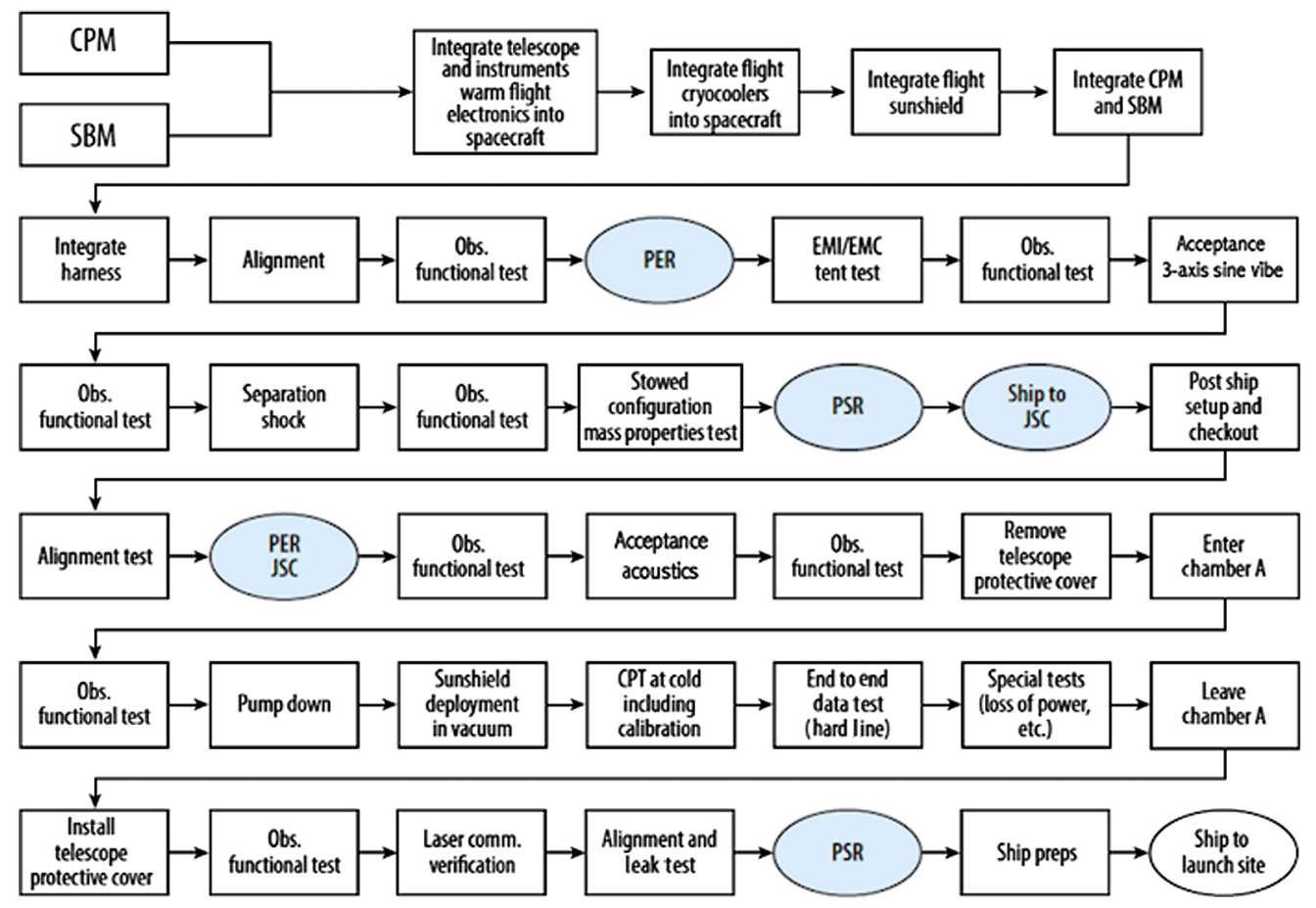

Fig. 22 Observatory I\&T summary flow. It includes the milestones reviews: PER and PSR.

\section{Launch Site Operations}

Prior to the arrival of the observatory, the GSE is housed in trailers or equipment shelters that are shipped to the launch site. The GSE is then tested to verify proper function and prepared to be utilized for final tests. The observatory is shipped to the launch site in its launch configuration. Functional tests must still be performed to ensure that transportation and handling activities have not adversely affected the observatory. It is important that any problems be identified and corrected before the next level of integration. These standalone activities are performed in a dedicated off-pad processing facility at the launch site. Any additional integration or testing activities are also performed during this phase. These can involve required corrective actions resulting from postshipment test anomalies or tasks otherwise planned to be completed just prior to launch (e.g., alignment, propulsion functional, and leakage tests). Origins will follow typical launch site operations that are described in the flow shown in Fig. 23. 


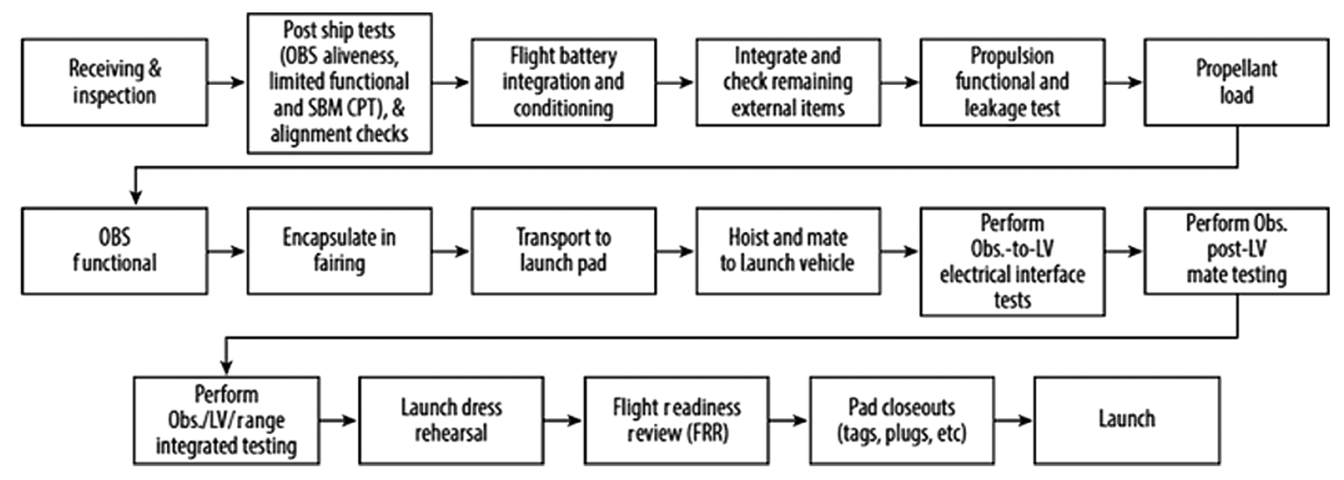

Fig. 23 Origins top level flow for launch site operations. OBS stands for observatory. LV stands for launch vehicle.

\section{Conclusions}

The CPM and the observatory I\&T planning and flow will benefit from using JWST documentation, metrology, tools, and lessons learned during I\&T implementation. The large size of the deployed sunshield combined with extreme temperature difference from the hot to cold zones makes thermal testing of the full observatory impractical. Instead, the cold zone elements are processed through a separate protoflight test program with cryogenic thermal vacuum testing and the hot zone is processed through its own protoflight test program that is analogous to a typical spacecraft I\&T flow. Many testbeds and simulators will be implemented to address cost and schedule risk and interface verification for this mission. Facilities to perform the I\&T program already exist and are owned by NASA.

\section{References}

1. "Origins Space Telescope study team's report to the 2020 Astrophysics Decadal Survey," https://asd.gsfc.nasa.gov/firs/docs/ (2019).

2. J. A. Corsetti et al., "Optical design of the Origins space telescope," Proc. SPIE 11115, 1111513 (2019).

3. D. Leisawitz et al., "The Origins Space Telescope," Proc. SPIE 11115, 111150Q (2019).

4. G. Jones and J. Marsh, "James Webb Space Telescope Integration \& Test," AIAA SPACE, Long Beach, California (2016).

Susanna Petro received her doctor's degree in physics from the University of Rome, Italy, and has more than 30 years of experience in spacecraft and instrument systems design, test, and launch. She is the NASA/GSFC Flight Systems Integration and Test branch staff engineer. She previously worked at the NASA Johnson Space Center and Thales Alenia Space in Europe in the development of satellites and payloads for telecommunications, science and exploration, and remote sensing. 